\title{
ListChecker Pro 1.2: A program designed to facilitate creating word lists using the University of South Florida word association norms
}

\author{
Deborah K. EAKIN \\ Mississippi State University, Mississippi State, Mississippi
}

\begin{abstract}
Various areas of research (e.g., memory, metamemory, visual word recognition, associative priming) rely on the careful construction of reliable word lists. ListChecker Pro 1.2 is a computer program that accesses the University of South Florida word association norms (Nelson, McEvoy, \& Schreiber, 1998, 2004) to report characteristics of words (e.g., frequency, concreteness), as well as direct and indirect associative relationships (e.g., shared associates, mediators). The present article presents the input requirements, menu options, and output obtained by ListChecker Pro 1.2. In addition, a randomly selected list of words from the associative versus semantic priming literature was submitted to ListChecker Pro 1.2 to demonstrate how seemingly unrelated words can be associated. The zipped file containing the program and database can be downloaded from www .eakinmemorylab.psychology.msstate.edu.
\end{abstract}

Knowledge about the characteristics of words and their relationships to each other is vital to several areas of research, including memory, metamemory, visual word recognition, and priming. The present article presents ListChecker Pro 1.2, a program designed to help researchers evaluate characteristics of constructed word lists. ListChecker Pro 1.2 accesses normative data represented in the University of South Florida (USF) word association norms (Nelson, McEvoy, \& Schreiber, 1998, 2004) to determine characteristics and associative information about words. More information on the USF word association norms can be found at http://web.usf.edu/FreeAssociation and in the online supplement for Nelson et al. (2004). The USF norms report characteristic data, such as printed word frequency and concreteness. In addition, the USF norms provide an index of direct and indirect associations among and between words. In essence, the USF norms provide a theoretical structure for the organization of words and their associative relationships (Nelson, McEvoy, \& Schreiber, 1998, 2004). ListChecker Pro 1.2 accesses this information and provides reports on the associative relationships among and between lists of words to either examine their impact experimentally (e.g., Eakin \& Hertzog, 2006; Nelson, Bennett, \& Leibert, 1997; Nelson, McEvoy, \& Dennis, 2000; Nelson, McEvoy, \& Pointer, 2003; Nelson, McEvoy, \& Schreiber, 1998, 2004; Nelson, McKinney, Gee, \& Janczura, 1998; Schreiber, 1998; Schreiber \& Nelson, 1998) or equate them across other experimental manipulations in order to control for the in- fluence of extraneous characteristics (e.g., Eakin, 2005; Hertzog, Kidder, Powell-Moman, \& Dunlosky, 2002).

A discussion of the types of research for which ListChecker Pro 1.2 facilitates list construction is presented. In addition, the ListChecker Pro 1.2 program is discussed here in detail, and example output is included for each option. First, however, a brief discussion of the types of information provided by the USF norms is provided.

\section{Associative Characteristics Provided by the USF Word Association Norms}

Consider a list of words that includes BOY, MOTHER, CHILD, FAST, RAKE, HAPPY, and RUN. According to the USF word association norms (Nelson, McEvoy, \& Schreiber, 1998, 2004), words on this list have both explicit direct associations and implicit indirect associations. Direct associations can be forward, from the presented word to its associates, or backward, from the associate to the presented word. Figure 1 illustrates some of the associations among words on the list. For instance, CHILD and MOTHER have a forward association strength of .03 and a backward association strength of $.01 .^{1}$ Direct associations are identified in the USF norms as members of a word's associative set. For example, CHILD has an associative set of 15 , whereas HAPPY has a relatively small associative set of 8 . The association between a word and its set member is a forward association; however, words can serve both as having an associative set and being a member of another

D. K. Eakin, deakin@psychology.msstate.edu 


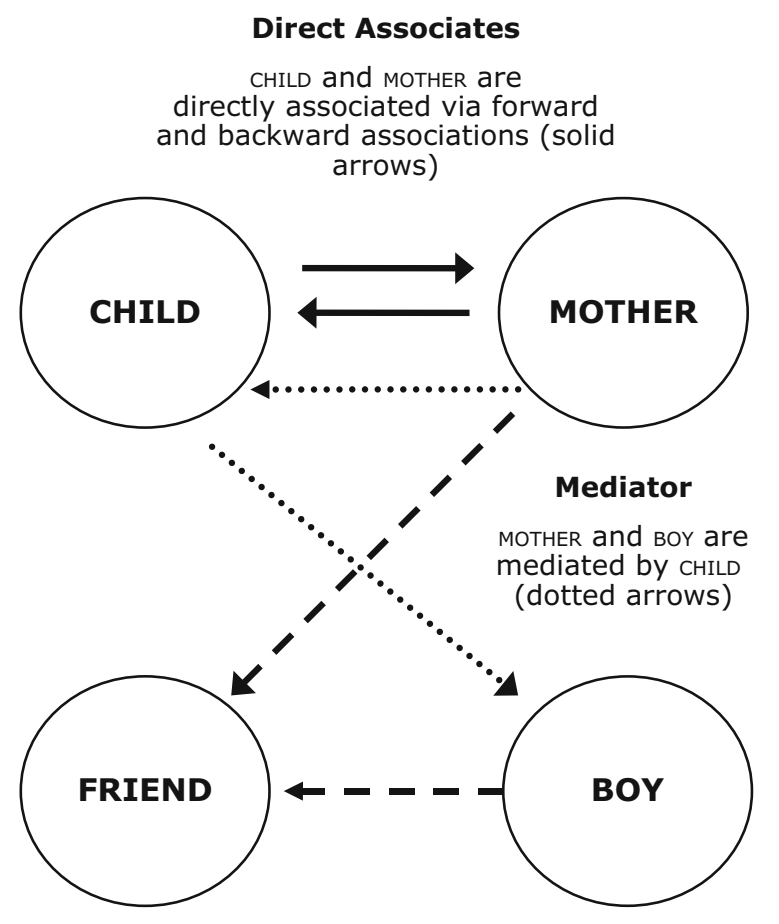

\section{Shared Associate}

MOTHER and BOY are associated via PARENT

(dashed arrows)

Figure 1. Schematic of potential associative relationships among words.

word's associative set. In such a case, the backward association is an indication that a word is also a member of the associative set of the word with which it shares a forward association.

Related to set size are two other characteristics of words: resonance and connectivity. Resonance refers to the resonant connections from the associates of the word back to the word itself. For example, FRIEND has a large associative set of 22 words, and, when each of those words was separately normed, 15 of them produced FRIEND as an associate, with a total resonance of 5.16. This value is calculated by taking the sum of the association strengths of those 15 members of FRIEND's set that produced it as an associate. Connectivity refers to the connections among the associates of the word. For example, the 22 associates of FRIEND, when separately normed, produced 41 associateto-associate connections. ${ }^{2}$

In addition to having direct associations, words can be indirectly associated through mediators and shared associates (formerly called overlapping associates). A mediator is a link between words, often associating words that are not directly associated. For instance, although MOTHER and BOY are not directly associated, they are mediated by CHILD, with a strength of .0003 (see note 2). MOTHER and BOY are also indirectly associated via a shared associate. Shared associates are associated with both the cue and the target, linking the two, even though they may not be directly related or mediated. FRIEND is a shared associa- tion of MOTHER and BOY with a shared associate strength of .0003. Note that, although FRIEND is not on the original list, because it shares associations with words that are on the list, it is implicitly present. In fact, FRIEND not only has a large associative set of 22 , it is also a member of the associative sets of over 100 words, including some of those on the list. Therefore, FRIEND has the potential to influence experiment outcomes, as will be discussed.

\section{How Word Association Influences \\ Experiment Outcomes}

The strength of direct and indirect connections between and among the associates of words, as well as the number of words, or set size, of a word, influences memory outcome (e.g., Eakin \& Hertzog, 2006; Nelson \& McEvoy, 1979; Nelson, McEvoy, \& Schreiber, 1990, 1998; Nelson, Schreiber, \& McEvoy, 1992; Nelson, Schreiber, \& Xu, 1999; Schreiber, 1998). In addition, both resonance and connectivity affect memory similarly; increased resonance and connectivity result in better memory (Nelson et al., 2003). Nelson and Zhang (2000) conducted a simultaneous multiple regression analysis of 29 experiments to determine whether the effects of associative characteristics affected recall. When the correlations among the variables were statistically controlled, each variable served as a significant predictor of recall, and nearly $50 \%$ of the variance in cued recall was explained by direct and indirect associations, target connectivity, and the set sizes of the cues and targets. Metamemory or knowledge about one's own memory (Flavell, 1979) is influenced by the number of associates of words in the same way that memory is influenced (e.g., Eakin \& Hertzog, 2006, 2010a; Hertzog et al., 2002; Schreiber, 1998; Schreiber \& Nelson, 1998; but see Eakin \& Hertzog, 2010b). However, memory and metamemory are not the only domains for which consideration of word associations is important.

The number and type of associates also influence visual word recognition. For instance, lexical decision is faster for pseudohomophones (nonwords that sound like an actual word; e.g., NALE) with small sets versus those with large sets (Yates, Locker, \& Simpson, 2003). In addition, knowledge about the relationships between words is crucial for research on semantic versus associative priming (for reviews, see Hutchinson, 2003; Lucas, 2000; Neely, 1991). Semantic priming is an experimental technique demonstrating that response time to a target word is faster if it is preceded by a related word, or prime, than if it is preceded by an unrelated word. For example, response time to indicate that DOCTOR is a word is faster when that word is primed with NURSE than when it is primed with TRUCK. Such research is useful for the understanding of how words are organized and accessed (e.g., Collins \& Loftus, 1975; McClelland, 1987; Plaut \& Booth, 2000). However, historically, word lists used in semantic priming research were created using word association norms, resulting in confounding between associative and semantic priming. Fischler (1977) made a distinction between semantic and associative priming by indicating that words could be related semantically but not associatively (e.g., NURSE-WIFE) and vice versa; he described the two types 
Table 1

Sample Word List Used As Input for ListChecker Pro 1.2

\begin{tabular}{ccc}
\hline $\begin{array}{c}\text { Cue-Target Stimuli List } \\
\text { (Also, Option 5 Input List) }\end{array}$ & $\begin{array}{c}\text { Cues } \\
\text { (Option 1, 2 Input List) }\end{array}$ & $\begin{array}{c}\text { Targets } \\
\text { (Option 1, 2 Input List) }\end{array}$ \\
\hline TIGER-CAT & TIGER & CAT \\
WARRIOR-SOLDIER & WARRIOR & SOLDIER \\
JOKE-LAUGH & JOKE & LAUGH \\
THIMBLE-NEEDLE & THIMBLE & NEEDLE \\
CIRCLE-ROUND & CIRCLE & ROUND \\
FILM-CAMERA & FILM & CAMERA \\
TAVERN-PUB & TAVERN & PUB \\
SOCKET-PLUG & SOCKET & PLUG \\
MOB-GANG & MOB & GANG \\
REPTILE-LIZARD & REPTILE & LIZARD \\
\hline
\end{tabular}

Note-The first column is the potential stimuli list. Option 5 could also be applied to this list. The second and third columns split the cue-target list into separate cue and target input lists. Both Options 1 and 2 could be applied to these lists.

of relationships as being mutually exclusive. Conversely, Steyvers and Tenenbaum (2005) have shown that the average associative path length to get from any one word to any other in the USF norms is three steps, thereby rendering the concept of completely unassociated words suspect. In fact, the USF word association norms indicate that NURSE and WIFE are actually associated via WOMAN. Research on priming frequently uses word lists that contain multiple members of the same taxonomic category (e.g., FATHER, GRANDFATHER-Pécher, Zeelenberg, \& Raaijmakers, 1998; and JACKET, TROUSER-Williams, 1996). For example, McRae and Boisvert (1998) demonstrated semantic priming of (presumably) unassociated words (e.g., GOOSE-CHICKEN). Association was determined via norming tasks conducted by the researchers. For words that were semantically similar but not associated, they reported priming effects, especially if the prime and target were highly similar. However, ListChecker Pro 1.2, using information from the USF word association norms, demonstrates that GOOSE and CHICKEN not only are directly associated (albeit weakly) but also are associated via five other words. The purpose of the present article is not to enter the debate on semantic versus associative priming but to demonstrate the importance of using a normative database - specifically the USF word association norms - to examine such factors and to introduce a program that facilitates reporting the characteristics of words in that database.

Although the USF word association norms are an excellent resource, using them to verify associative relationships among and between words on a list can be cumbersome. The purpose of the present article is to describe ListChecker Pro 1.2, a computer program designed to use the normative information represented by the USF word association norms to create accurate word lists for experimental purposes.

\section{LISTCHECKER PRO 1.2}

ListChecker Pro 1.2 is a program that consists of an application executable file and a data file containing information from Appendix A of the USF word association norms (see Nelson et al., 2004, for a description of all of the appendixes). The program is Windows based and performs five operations; these options are described in the following sections. Instructions for running the program are given in a "read me" file attached with the program, although some general points are discussed in the Method section of the present article. Table 1 presents sample input lists for Options 1, 2, and 5; these lists were used in the examples presented throughout this section.

\section{Option 1: Compare Two Lists of Single Words \\ for Shared Associates}

Option 1 allows for the comparison of two lists of any length (even a single word) to determine the relationships between the words on the first list and the words on the second list. Option 1 could be used, for example, to construct a list of related cue-target pairs for paired-associate learning, intralist cuing, or priming tasks. Assume that the goal is to create a list of cue-target pairs for which the cue is related to only its intended target. Option 1 could determine the direct and indirect relationships between the cue list and the target list. In addition, Option 1 could show the shared associates and mediators for each set of related pairs. The list of cues serves as the first input list, and the list of targets serves as the second input list. The output file that is created by ListChecker Pro 1.2 is a semicolondelimited text file that can be opened in Microsoft Excel. A sample output of the comparison done by Option 1 is shown in Figure 2. Each cue is listed in column A, followed by its set size in column B. In column $\mathrm{C}$, the intended target is listed along with the other targets from List 2 for which there are direct and indirect associations with the List 1 cue. The corresponding set sizes are listed in column $\mathrm{D}$. The number of direct associations can be determined from looking at columns $\mathrm{E}$ and $\mathrm{F}, \mathrm{G}$ and $\mathrm{H}$, and I. Column E lists the forward association strength between the cue (e.g., TIGER) and the target listed (e.g., CAT); column F places a " 1 " in that column to serve as a count of one target for which there is a forward association strength greater than zero. Columns $\mathrm{G}$ and $\mathrm{H}$ provide the same information regarding any backward association strength from the target to the cue, as well as a count-mark, indicat- 

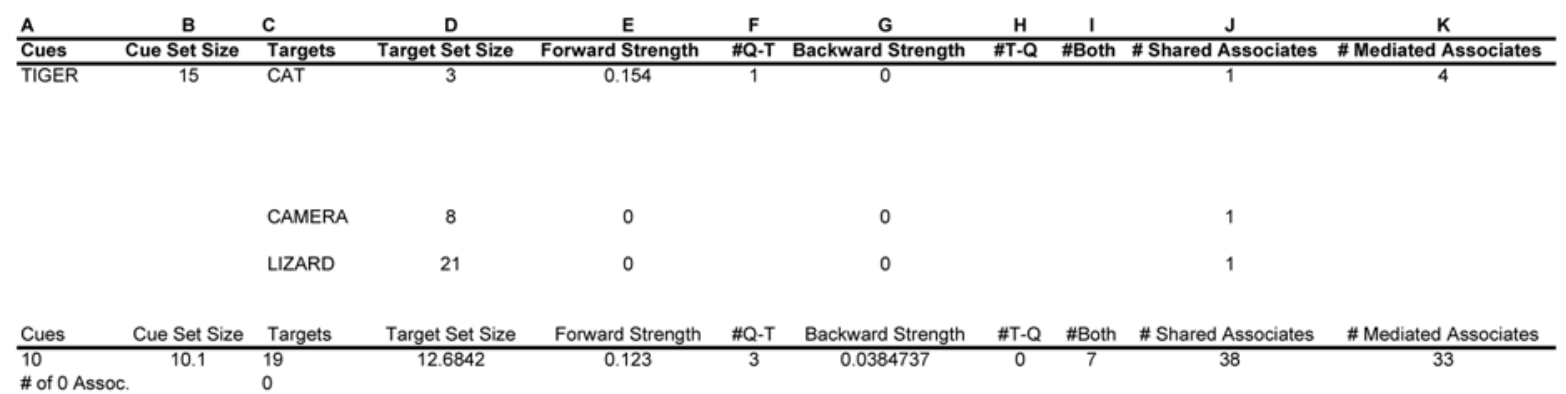

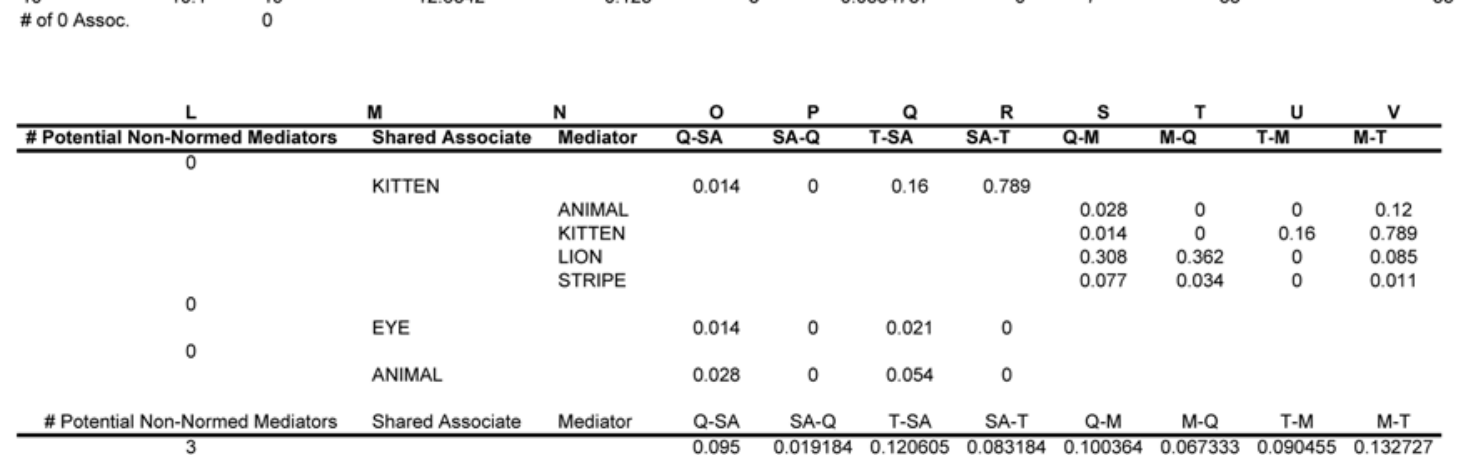

Figure 2. Sample output from Option 1. This figure is a snapshot of the actual output from Option 1 for which the list of cues and list of targets are compared with each other to determine associative relationships among words on the lists. The output is truncated and split for optimal presentation. The bottom rows indicate the total means for all characteristics for all words in the output.

ing that association. Column I provides a count-mark indicating both forward and backward associations between the cue and target - the strengths are listed in columns E and $\mathrm{G}$, respectively.

In addition to being directly associated, TIGER and CAT are also indirectly associated via one shared associate and four mediators. Column J indicates a count of the number of shared associates; column $\mathrm{K}$ indicates a count of the number of mediators. Column $\mathrm{J}$ indicates the number of potential nonnormed mediators: It is a count of words that were provided as responses to a list item but that have not been submitted to norming themselves. Therefore, they could be mediators, but, as yet, have not been normed. This number is important, because a large number of nonnormed mediators increases the likelihood of underestimating mediator strength. A count for potential nonnormed shared associates is not provided, because nonnormed shared associates do not have an impact on the shared association strength (Nelson, McKinney, et al., 1998). Columns M and N list the shared associate and mediator, respectively. The next eight columns provide the forward and backward association strengths between the cue or target and the shared associate (columns $\mathrm{O}-\mathrm{R}$ ) and between the cue or target and the mediator (columns $\mathrm{S}-\mathrm{V}$ ). Information about the indirect associations is provided both for targets with a direct association to the cue - such as for the TIGER-CAT pair-but also for targets for which only indirect associations exist. For example, TIGER is not directly associated with LIZARD, but the two words are indirectly associated via a shared associate, ANIMAL.
Finally, the last row of the output from Option 1 provides the sums of the count columns and the means of the value columns for each of the characteristics described, in addition to a count of cues and/or targets on the list that have not been normed. (Note that the totals are from the whole report: The report in Figure 2 is truncated to save space.)

\section{Option 2: Compare One Single List of Words With Itself for Shared Associates}

Option 2 compares each word on a list of single words with every other word on the list, and the output describes the relationships among words on the list. Optimally, list criteria would not only require that each cue relate only to its intended target, they would also require that each cue should not be related to another cue word on the list. Option 2 allows for an examination of the relationships among all of the cues or among all of the targets. The input text file would consist of just the list of cues or targets from Table 1. The output file is a semicolondelimited text file that can be opened in Excel. Figure 3 shows sample output resulting from Option 2. The column headings and values are the same as those for Option 1. Each cue on the list is compared with every other cue. Although there are no direct associations among the cues, note that three cues are indirectly associated via shared associates (e.g., SOCKET and TIGER are indirectly associated via EYE). The last row of the output from Option 2 provides the sums of the count columns and the means of the value columns for each of the characteristics described, as well as a count of the words on the list that have not been normed. 


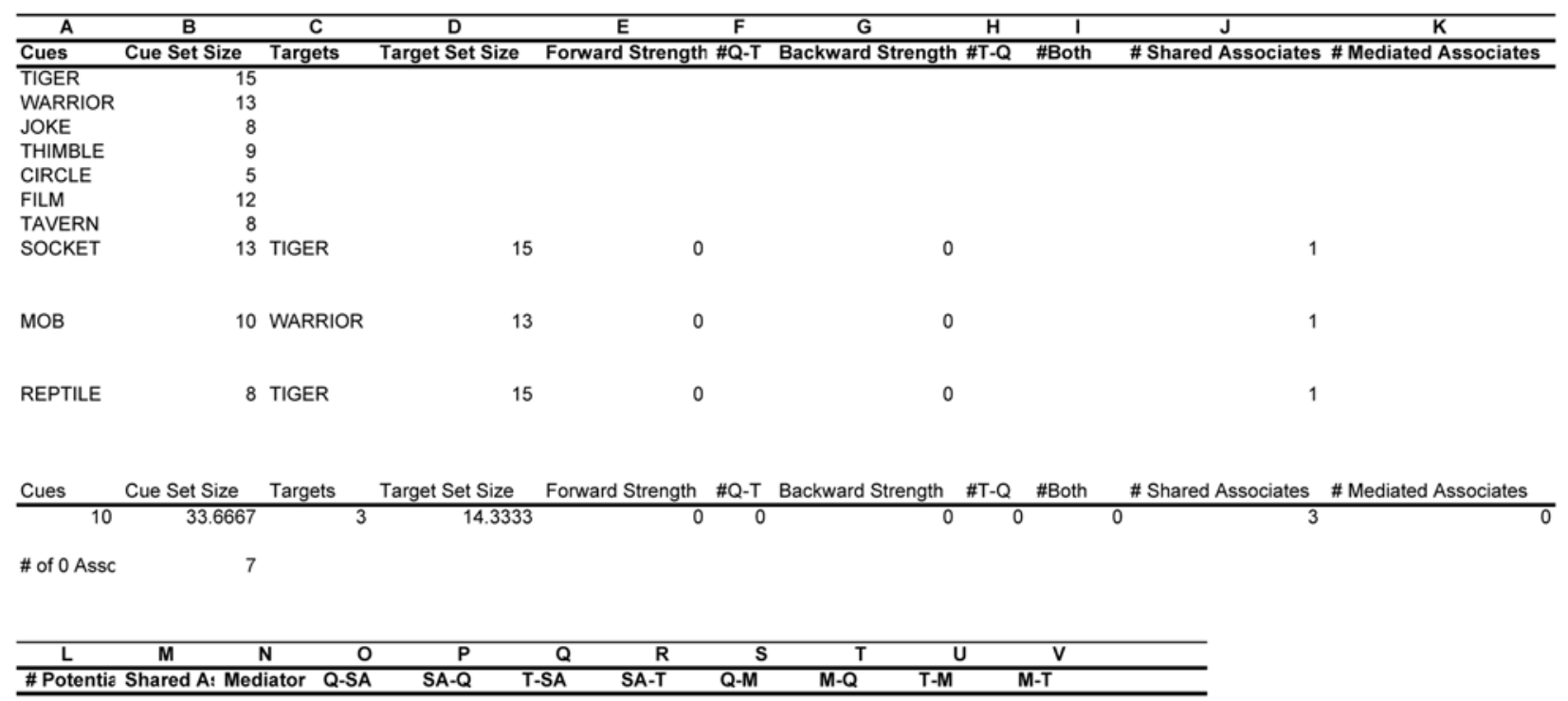

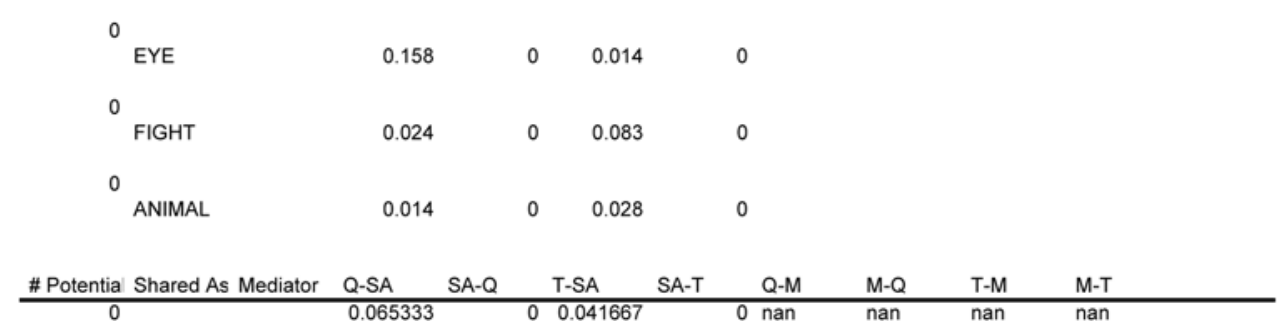

Figure 3. Sample output from Option 2. Note that SOCKET is compared with other cues from the cue input list (e.g., TIGER).

\section{Options 3 and 4: Get Characteristics of Words}

Option 4 takes, as input, a list of single words, and the output describes the characteristics of each word on the list. The output is a semicolon-delimited text file that can be opened in Excel. The characteristics of each word include the sample size, set size, frequency, concreteness, connectivity strength, mean connectivity, part of speech, and resonance (see Figure 4). The output also shows a count of words on the list that have not been normed. For Option 3, a single word can be entered at the command prompt, and the characteristics of that word are displayed on the screen.

\section{Option 5: Get Cue/Response Relationships}

Option 5 takes as input a list consisting of cue-target pairs; the pairs are listed in a text file, in capital letters, separated by a space (see the first column in Table 1). The output text file not only contains the characteristics of each word described in Options 3 and 4, but also displays the direct and indirect associative relationships between the cue (column A) and target (column J; see Figure 5). Therefore, not only are the sample size, set size, frequency, concreteness, connectivity strength, mean connectivity, part of speech, and resonance displayed for the cue (columns B-I) and for the target (columns K-R), but the forward and backward association strengths are also displayed (columns $\mathrm{S}$ and $\mathrm{T}$ ). Indirect associations, including the number of mediators and shared associates, are displayed in columns $\mathrm{U}$ and $\mathrm{V}$, respectively. However, Option 5 does not present other information from Option 1 , such as the number of associations between cues and other targets on the list: Only associates between the intended cue-target pair are listed in the Option 5 output. Option 5 provides paired associate information in a convenient table format, to facilitate computation of means in order to compare means across two lists, for instance.

\section{Analysis of List Used in \\ McRae and Boisvert (1998)}

As previously mentioned, participants' knowledge about words can have an impact on experimental manipulations that are intended to be episodic to the experiment (Nelson et al., 2003; Nelson, McKinney, et al., 1998; Nelson et al., 1992). One such domain that depends on independence between the experimental manipulation and prior knowledge is that of semantic versus associative priming tasks. At issue is whether priming can be observed in the absence of association between the prime and the target (for reviews, see Hutchinson, 2003; Lucas, 2000; Neely, 1991). Many studies have reported semantic priming of unassociated 


\begin{tabular}{|c|c|c|c|c|c|c|c|c|}
\hline $\mathbf{A}$ & B & c & D & E & $\mathrm{F}$ & G & $\mathrm{H}$ & 1 \\
\hline Cues & Sample Size & Cue Set Size & Cue Frequency & Cue Concreteness ( & Cue Connectivity Strength & Mean Cue Connectivity & Cue Part of Speech & Cue Resonance Probability \\
\hline$\overline{\text { TIGER }}$ & 143 & 15 & 7 & 6.07 & 3.17 & 1.13 & 0 & 0.2 \\
\hline WARRIOR & 145 & 13 & 5 & 5.13 & 2.51 & 1.92 & 0 & 0.25 \\
\hline JOKE & 165 & 8 & 22 & 3.48 & 2.06 & & $\mathrm{~N}$ & 0.57 \\
\hline THIMBLE & 156 & 9 & 1 & 5.18 & 4.13 & 2.33 & 0 & 0 \\
\hline CIRCLE & 156 & 5 & 60 & 5.14 & 1.84 & & $\mathrm{~N}$ & 1 \\
\hline FILM & 138 & 12 & 96 & 5.91 & 4.08 & & $\mathrm{~N}$ & 0.55 \\
\hline TAVERN & 147 & 8 & 2 & 6.18 & 3.29 & 1.5 & 0 & 0.13 \\
\hline SOCKET & 139 & 13 & 3 & 5.9 & 3.48 & 1.62 & $\mathrm{~N}$ & 0.31 \\
\hline MOB & 127 & 10 & 10 & 5.1 & 2.58 & & $\mathrm{~N}$ & 0.4 \\
\hline REPTILE & 148 & 8 & 0 & 6.65 & 1.58 & 1.5 & $\mathrm{~N}$ & 0.63 \\
\hline
\end{tabular}

Figure 4. Sample output from Option 4, which provides characteristics of a list of words.

prime-target pairs (see Lucas, 2000, for a review). The purpose of the present experiment was to examine a list of presumably unassociated prime-target word pairs to determine whether-according to ListChecker Pro 1.2 and the USF word association norms - the word pairs were unassociated.

McRae and Boisvert (1998) was selected at random from among the studies cited in the Lucas (2000) review article for which the word lists were available. One stated goal of McRae and Boisvert was to demonstrate "the existence of automatic semantic similarity priming in the absence of normative association" (p. 558). They compared priming effects between a list of similar and dissimilar primes for 38 targets (Experiment 1): The targets were divided into two lists of 19 targets each. McRae and Boisvert conducted pilot studies, both to select targets that were not associatively related and to rule out both forward and backward prime-target associations. One pilot study required college students to make judgments of association; another used a technique similar to that used to create the USF word association norms. Students were provided with a word and were asked to generate an associate.

\section{METHOD}

\section{Materials}

The sample word lists were taken from McRae and Boisvert (1998, Experiment 1) and are listed in Table 2. The comparison lists were their similar primes (SPs) and targets (Ts). Because the dissimilar primes are not relevant to our study, these lists were not included in the analyses. Separate text files were created for each list $(1,2)$ and each list type (SP, T), resulting in four input files: SP1, $\mathrm{SP} 2, \mathrm{~T} 1$, and $\mathrm{T} 2$.

\section{Procedure}

Comparing words within each list. A comparison of words within each list is important because providing a prime that is as-

\begin{tabular}{|c|c|c|c|c|c|c|c|c|}
\hline A & \begin{tabular}{l|} 
B \\
\end{tabular} & C & D & E & $\mathbf{F}$ & G & $\mathbf{H}$ & 1 \\
\hline Cues & Cue Sample Size & Cue Set Size & Cue Frequency & Cue Concreteness & Cue Connectivity Strength & Mean Cue Connectivity & Cue Part of Speech & Cue Resonance Probability \\
\hline TIGER & 143 & 15 & 7 & 6.07 & $\begin{array}{r}3.17 \\
\end{array}$ & $1.13 \mathrm{~N}$ & & $\begin{array}{r}0.2 \\
\end{array}$ \\
\hline WARRIOR & 145 & 13 & 5 & 5.13 & 2.51 & $1.92 \mathrm{~N}-\mathrm{N} \mathrm{r}-\mathrm{s}$ & & 0.25 \\
\hline JOKE & 165 & 8 & 22 & 3.48 & 2.06 & $1 N$ & $\mathrm{~N}$ & 0.57 \\
\hline THIMBLE & 156 & 9 & 1 & 5.18 & 4.13 & $2.33 \mathrm{~N}$ & & 0 \\
\hline CIRCLE & 156 & 5 & 60 & 5.14 & 1.84 & $1 \mathrm{~N}$ & $\mathrm{~N}$ & 1 \\
\hline FILM & 138 & 12 & 96 & 5.91 & 4.08 & $2 \mathrm{~N}$ & $\mathrm{~N}$ & 0.55 \\
\hline TAVERN & 147 & 8 & 2 & 6.18 & 3.29 & $1.5 \mathrm{~N}$ & & 0.13 \\
\hline SOCKET & 139 & 13 & 3 & 5.9 & 3.48 & $1.62 \mathrm{~N}$ & & 0.31 \\
\hline MOB & 127 & 10 & 10 & 5.1 & 2.58 & $2 \mathrm{~N}$ & & 0.4 \\
\hline REPTILE & 148 & 8 & 0 & 6.65 & 1.58 & $1.5 \mathrm{~N}$ & & 0.63 \\
\hline
\end{tabular}

\begin{tabular}{|c|c|c|c|c|c|c|c|}
\hline $\mathrm{J}$ & \begin{tabular}{r|}
$\mathbf{K}$ \\
\end{tabular} & $\mathrm{L}$ & $\mathrm{M}$ & $\mathrm{N}$ & 0 & $\mathbf{P}$ & $\mathbf{Q}$ \\
\hline Targets & Target Sample Size & Target Set Size & Target Frequency & Target Concreteness(!) & Target Connectivity Strength & Mean Target Connectivity & Target Part of Speech \\
\hline CAT & 155 & 3 & 23 & 6.21 & 0.93 & & $\mathrm{~N}$ \\
\hline SOLDIER & 150 & 13 & 39 & 5.68 & 2.18 & 2 & $\mathrm{~N}$ \\
\hline LAUGH & 165 & 8 & 28 & 4.29 & 1.95 & 1.75 & v \\
\hline NEEDLE & 165 & 8 & 15 & 5.79 & 2.17 & 2.12 & $\mathrm{~N}$ \\
\hline ROUND & 127 & 7 & 81 & 4.34 & 1.98 & 0.43 & AJ \\
\hline CAMERA & 145 & 8 & 36 & 6.23 & 1.67 & 0.71 & $\mathrm{~N}$ \\
\hline PUB & 139 & 6 & 1 & 6.05 & 2.83 & 3 & $\mathrm{~N}$ \\
\hline PLUG & 144 & 25 & 23 & 6 & 3.67 & 1.3 & $\mathrm{~N}$ \\
\hline GANG & 132 & 18 & 22 & 5.55 & 2.18 & 1.33 & $\mathrm{~N}$ \\
\hline LIZARD & 149 & 21 & 1 & 5.67 & 4.13 & 2.17 & $\mathrm{~N}$ \\
\hline
\end{tabular}

\begin{tabular}{crrrr|}
$\mathbf{R}$ & $\mathbf{S}$ & $\mathbf{T}$ & $\mathbf{U}$ & $\mathrm{V}$ \\
\hline Target Resonance Probability & Forward Strength & Backward Strength & \#Shared Associates & \#Mediated Associates \\
\hline 1 & 0.154 & 0 & 1 & 4 \\
0.42 & 0.186 & 0.033 & 5 & 2 \\
0.88 & 0.612 & 0.055 & 1 & 3 \\
0.88 & 0.218 & 0 & 5 & 4 \\
0.57 & 0.199 & 0.197 & 1 & 3 \\
0.71 & 0.116 & 0.166 & 2 & 2 \\
0.33 & 0.259 & 0 & 3 & 2 \\
0.3 & 0.173 & 0.111 & 4 & 3 \\
0.22 & 0.15 & 0.015 & 3 & 3 \\
0.22 & 0.27 & 0.154 & 4 & 2
\end{tabular}

Figure 5. Sample output from Option 5, which provides characteristics of a list of word pairs, including associative characteristics. 
Table 2

Sample Word Lists (From McRae \& Boisvert, 1998 , Experiment 1) Submitted to ListChecker Pro 1.2's Options 1, 2, and 5

\begin{tabular}{|c|c|c|}
\hline Cues (SP1) & Targets (T1) & Cue-Target Pairs (List 1) \\
\hline PARAKEET* & BUDGIE* & PARAKEET-BUDGIE \\
\hline $\mathrm{FINCH}^{*}$ & CANARY & FINCH-CANARY \\
\hline GOOSE & TURKEY & GOOSE-TURKEY \\
\hline EAGLE & HAWK & EAGLE-HAWK \\
\hline DUCK & CHICKEN & DUCK-CHICKEN \\
\hline WHALE & DOLPHIN & WHALE-DOLPHIN \\
\hline MOOSE & CARIBOU* & MOOSE-CARIBOU \\
\hline PLUM & PRUNE & PLUM-PRUNE \\
\hline COCONUT & PINEAPPLE* & COCONUT-PINEAPPLE \\
\hline RADISH & BEETS & RADISH-BEETS \\
\hline PUMPKIN & SQUASH & PUMPKIN-SQUASH \\
\hline PEAS & BEANS & PEAS-BEANS \\
\hline SLIPPERS & SANDALS & SLIPPERS-SANDALS \\
\hline BRA & CAMISOLE* & BRA-CAMISOLE \\
\hline TIE & BELT & TIE-BELT \\
\hline LAMP & CHANDELIER $^{*}$ & LAMP-CHANDELIER \\
\hline CLOSET & DRESSER & CLOSET-DRESSER \\
\hline CUSHION & PILLOW & CUSHION-PILLOW \\
\hline MAT & CARPET & MAT-CARPET \\
\hline Cues (SP2) & Targets (T2) & Cue-Target Pairs (List 2) \\
\hline MICROWAVE & TOASTER & MICROWAVE-TOASTER \\
\hline BOTTLE & JAR & BOTTLE-JAR \\
\hline CRAYON & PENCIL & CRAYON-PENCIL \\
\hline HOE & SHOVEL & HOE-SHOVEL \\
\hline FILE & SANDPAPER & FILE-SANDPAPER \\
\hline SHED & BARN & SHED-BARN \\
\hline YACHT & SHIP & YACHT-SHIP \\
\hline CANOE & RAFT & CANOE-RAFT \\
\hline BUS & SUBWAY & BUS-SUBWAY \\
\hline WAGON & CART & WAGON-CART \\
\hline TRUCK & VAN & TRUCK-VAN \\
\hline JEEP & DUNEBUGGY* & JEEP-DUNEBUGGY \\
\hline MOTORCYCLE & SCOOTER $^{*}$ & MOTORCYCLE-SCOOTER \\
\hline $\mathrm{AXE}^{*}$ & TOMAHAWK ${ }^{*}$ & AXE-TOMAHAWK \\
\hline MISSILE* & BOMB & MISSILE-BOMB \\
\hline SLINGSHOT* & CATAPULT* & SLINGSHOT-CATAPULT \\
\hline RIFLE & PISTOL & RIFLE-PISTOL \\
\hline CANNON & BAZOOKA $^{*}$ & CANNON-BAZOOKA \\
\hline SWORD & SPEAR & SWORD-SPEAR \\
\hline
\end{tabular}

${ }^{*}$ Nonnormed words.

sociated with another prime could interfere with priming effects on the intended target. Likewise, a target should not serve as a similar prime for another target. To determine whether primes and targets were associated with each other, each of the individual similar prime (SP1 and SP2) and target (T1 and T2) text files was submitted to Option 2 in ListChecker Pro 1.2 in order to determine whether items within a list could interfere with priming of the intended target.

Comparing prime lists with target lists. Each prime on the list should serve as a prime for only its intended target; a word should not be a potential similar prime for more than one target. To determine whether this constraint was achieved, each of the SP input files and their associated $\mathrm{T}$ files were submitted to Option 1 in ListChecker Pro 1.2, in order to determine three things: direct associations between the primes and their intended targets; direct associations between the primes and other, nonintended, targets; and indirect associations among the primes and targets. The reports generated from this option describe the specific prime-target relationships (however, see the Option 5 procedure, described next), but also generate the overall number of each of the three types of relationships listed. In addition, words that are implicated in indirect relationships are specified, as well as their association strength to each prime and target.
Determining associative relatedness for each prime-target pair. Each of the two text files that listed each prime-target pair for each list was submitted to Option 5 in ListChecker Pro 1.2. The goals were to determine whether direct relationships existed between intended prime-target pairs and the number and type of indirect relationships specific to each pair.

\section{RESULTS}

\section{Comparing Words Within Each List}

Each list (SP1, SP2, T1, and T2) was compared with itself by using Option 2. Among the similar primes and targets, there were four sets in which a prime or target was directly associated to another prime/target on its list. For instance, when SP1 was compared with itself, ListChecker Pro 1.2 revealed that DUCK and GOOSE had a forward association strength of .162 and a backward association strength of .261. In addition to direct associations, a number of indirect associations were revealed by the program: Across the four lists there were 122 shared associates, 40 mediators, and 15 potential nonnormed mediators (also known as missing in action [MIA] mediators). See Table 3 for a summary of direct and indirect associations for all comparisons.

\section{Comparing Prime Lists With Target Lists}

Each similar prime list (SP1, SP2) was compared with its intended target list $(\mathrm{T} 1, \mathrm{~T} 2)$ by using Option 1. Although, according to the operational definition for similar primes in the study, none of the primes were supposed to be associated with their intended targets (or presumably with any other target on the list), 21 intended prime-target pairs were directly associated. For example, CUSHION was a similar prime for PILLOw; the two had a forward association strength of .194 and a backward association strength of .03. In addition, four primes were directly associated with an unintended target. For instance, CANNON (whose intended target was BAZOOKA) was directly associated with SHIP (forward strength $=.02$ ) and with BOMB (forward strength $=$ .03 ). The number of indirect associations was striking: There were 174 shared associations between primes and targets (81 on List 1, 93 on List 2), as well as 57 mediators and 12 MIA-potential nonnormed mediators (see Table 3 ). The numbers of associations may be higher - two primes and five targets had not been normed.

\section{Determining Associative Relatedness \\ for Each Prime-Target Pair}

The Option 5 output specifies the direct forward and backward association strength for prime-target pairs, and sometimes identifies additional associations. This information could be calculated from the output from Option 1, but the Option 5 output organizes the association information divulged by ListChecker Pro 1.2 in a format that matches primes and their intended targets in a single row and also provides the word characteristics for each prime (cue) and target. Figure 6 shows the Option 5 output from the SP1 to T1 comparison. Note that associations between pairs can be quickly assessed. For instance, WHALEDOLPHIN not only had a forward association strength of 
Table 3

Summary of Direct and Indirect Association for All Comparisons

\begin{tabular}{|c|c|c|c|c|c|c|c|}
\hline \multirow[b]{2}{*}{ Comparison } & \multirow[b]{2}{*}{ Option } & \multicolumn{3}{|c|}{ Direct Association } & \multicolumn{3}{|c|}{ Indirect Association } \\
\hline & & Forward & Backward & Both & Shared & Mediated & MIA Med \\
\hline $\mathrm{SP} 1 \times \mathrm{SP} 1$ & 2 & 1 & 1 & & 24 & 4 & 3 \\
\hline $\mathrm{SP} 2 \times \mathrm{SP} 2$ & 2 & 1 & 1 & & 54 & 24 & 8 \\
\hline $\mathrm{T} 1 \times \mathrm{T} 1$ & 2 & 1 & 1 & & 28 & 8 & 4 \\
\hline $\mathrm{T} 2 \times \mathrm{T} 2$ & 2 & 0 & 0 & & 16 & 4 & \\
\hline \multirow[t]{2}{*}{$\mathrm{SP} 1 \times \mathrm{T} 1$} & 1 & $\begin{array}{l}1 \\
1^{*}\end{array}$ & 2 & 6 & 81 & 31 & 7 \\
\hline & 5 & 2 & 3 & 5 & 28 & 12 & \\
\hline \multirow[t]{3}{*}{$\mathrm{SP} 2 \times \mathrm{T} 2$} & 1 & 4 & 2 & 6 & 93 & 26 & 5 \\
\hline & & $2^{*}$ & $2^{*}$ & & & & \\
\hline & 5 & 4 & 4 & 6 & 35 & 12 & \\
\hline
\end{tabular}

${ }^{*}$ Direct association with unintended target.

.031 and a backward association strength of .05 , it also shared five associates and had one mediating associate. (The output from Option 1 indicates that the shared associates are FISH, OCEAN, MAMMAL, WATER, and SAVE; the mediator is MAMMAL.)

\section{Comparison of Dissimilar Prime} and Target Lists

As a comparison, the lists of dissimilar primes were compared with themselves and with their intended targets. For the prime-to-prime comparison, only one prime

\begin{tabular}{|c|c|c|c|c|c|c|c|c|}
\hline A & B & c & D & E & $\mathbf{F}$ & G & H & 1 \\
\hline Cues & Cue Sample Size & Cue Set Size & Cue Frequency & Cue Concreteness & Cue Connectivity Strength & Mean Cue Connectivity & Cue Part of Speech & Cue Resonance Probability \\
\hline DUCK & 148 & 16 & 9 & 5.68 & 2.27 & 1.69 & $\mathrm{~N}$ & 0.39 \\
\hline EAGLE & 148 & 11 & 5 & 5.63 & 2.67 & 1.09 & $\mathrm{~N}$ & 0.27 \\
\hline \multirow{2}{*}{\multicolumn{9}{|c|}{$\begin{array}{l}\text { PARAKEET } \\
\text { FINCH }\end{array}$}} \\
\hline \multicolumn{3}{|l|}{ FINCH } & & & & & & \\
\hline GOOSE & 165 & 11 & 4 & 6.63 & 2.44 & 1.82 & $\mathrm{~N}$ & 0.46 \\
\hline WHALE & 97 & 17 & 0 & 6.23 & 2.96 & 1.73 & $\mathrm{~N}$ & 0.4 \\
\hline MOOSE & 165 & 18 & 0 & 6.03 & 4.96 & 2.17 & $\mathrm{~N}$ & 0.22 \\
\hline PLUM & 127 & 13 & 1 & 6.18 & 3.73 & 2.33 & $\mathrm{~N}$ & 0.33 \\
\hline \multicolumn{9}{|l|}{ COCONUT } \\
\hline RADISH & 146 & 14 & 8 & 0 & 2.46 & 1.85 & $\mathrm{~N}$ & 0.08 \\
\hline PUMPKIN & 140 & 8 & 2 & 6.42 & 0.96 & 0.12 & $\mathrm{~N}$ & 0.5 \\
\hline PEAS & 148 & 13 & 24 & 0 & 0 & & $\mathrm{~N}$ & 0 \\
\hline SLIPPERS & 119 & 8 & 7 & 5.74 & 2.92 & 0.88 & $\mathrm{~N}$ & 0.13 \\
\hline BRA & 140 & 15 & 0 & 6.25 & 0 & & $\mathrm{~N}$ & \\
\hline TIE & 148 & 17 & 23 & 5.55 & 2.19 & 1.14 & v & 0.64 \\
\hline LAMP & 156 & 7 & 18 & 6.09 & 2.09 & 0.71 & $\mathrm{~N}$ & 0.57 \\
\hline CLOSET & 147 & 14 & 16 & 5.95 & 2.23 & 1.38 & $\mathrm{~N}$ & 0.31 \\
\hline CUSHION & 139 & 12 & 8 & 0 & 4.61 & 2.92 & $\mathrm{~N}$ & 0.5 \\
\hline MAT & 148 & 12 & 5 & 5.02 & 2.13 & & $\mathrm{~N}$ & 0.33 \\
\hline
\end{tabular}

\begin{tabular}{|c|c|c|c|c|c|c|c|}
\hline $\mathrm{J}$ & \begin{tabular}{l|l}
$K$ & \\
\end{tabular} & \begin{tabular}{l|l}
$\mathbf{L}$ & $\mathrm{L}$
\end{tabular} & \begin{tabular}{l|l}
$M$ & \\
\end{tabular} & \begin{tabular}{l|l}
$\mathbf{N}$ & \\
\end{tabular} & $\begin{array}{ll}0 & 1 \\
\end{array}$ & \begin{tabular}{|l|}
$\mathbf{P}$ \\
\end{tabular} & Q \\
\hline Targets & Target Sample Size & Target Set Size & Target Frequency & Target Concreteness( & Target Connectivity Strength & Mean Target Connectivity & Target Part of Speech \\
\hline CHICKEN & 184 & 29 & 37 & 5.79 & 4.83 & & $\mathrm{~N}$ \\
\hline HAWK & 140 & 8 & 14 & 6.1 & 2.08 & 1.25 & $\mathrm{~N}$ \\
\hline BUDGIE & & & & & & & \\
\hline CANARY & 130 & 4 & 0 & 5.56 & 1.7 & 1.5 & $\mathrm{~N}$ \\
\hline TURKEY & 149 & 14 & 9 & 6.63 & 3.8 & 2.23 & $\mathrm{~N}$ \\
\hline DOLPHIN & 139 & 18 & 1 & 0 & 4.12 & & $\mathrm{~N}$ \\
\hline CARIBOU & & & & & & & \\
\hline PRUNE & 94 & 14 & 1 & 6.26 & 2.68 & 1.55 & $\mathrm{~N}$ \\
\hline PINEAPPLE & & & & & & & \\
\hline BEETS & & & & & & & \\
\hline SQUASH & 154 & 20 & 2 & 0 & 2.99 & 2.19 & $\mathrm{~N}$ \\
\hline BEANS & 150 & 21 & 9 & 6 & 0 & & $\mathrm{~N}$ \\
\hline SANDALS & 148 & 11 & 5 & 6.05 & 3.8 & 1.2 & $\mathrm{~N}$ \\
\hline CAMISOLE & & & & & & & \\
\hline BELT & 127 & 11 & 29 & 5.98 & 1.48 & 1.27 & $\mathrm{~N}$ \\
\hline CHANDELIER & & & & & & & \\
\hline DRESSER & 149 & 14 & 1 & 5.56 & 3.25 & 2.08 & $\mathrm{~N}$ \\
\hline PILLOW & 165 & 14 & 8 & 6.12 & 2.67 & 0.93 & $\mathrm{~N}$ \\
\hline CARPET & 145 & 17 & 13 & 5.68 & 3.55 & & \\
\hline
\end{tabular}

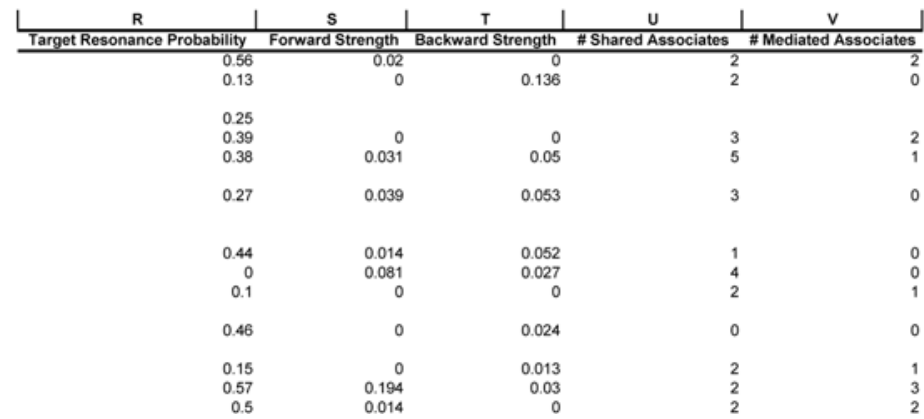

Figure 6. Output from Option 5 comparing SP1-T1 prime-target pairs from McRae and Boisvert (1998). 
had a direct forward and backward association (GOOSEDUCK $=.261$ and .162 , respectively), and only one prime had a backward association (TRUCK-JEEP $=.025$ ). There were 69 shared associates and 14 mediators revealed in the Option 2 comparisons and 107 shared associates and 26 mediators in the Option 1 comparison.

\section{DISCUSSION}

The importance of using normative data in creating word lists was demonstrated by the findings from the present experiment. Although McRae and Boisvert (1998) conducted some norming tasks to determine whether the semantically related words used in their studies were associated, the results demonstrate that their norms failed to reveal direct associations between prime-target pairs. However, even if there had been no direct associations between prime-target pairs, what McRae and Boisvert's norming task failed to identify were the myriad indirect associations between the prime-target pairs. Not only were there both direct and indirect associations within the prime and target lists, hundreds of associates either were shared between the prime-target pairs or mediated them. The seemingly extreme number of indirect associates begs the question as to whether a list could ever be created for which there were no direct and/or indirect associates. As a comparison, the lists of dissimilar primes were compared with themselves and with their intended targets; the numbers of associations were notably smaller than for the similar primes and their targets. Therefore, the concept proposed by McRae and Boisvert - that semantic similarity and not normative association produces the priming effects they obtained - is not possible to discern, given the analysis of their word lists by ListChecker Pro 1.2. However, the lists of dissimilar primes were far from being free of indirect associations. This finding is not surprising, given that the dissimilar lists were created by trading List 1 and List 2 primes, or by re-pairing similar primes with different targets on the same list. Therefore, because the same primes and targets were present on the dissimilar as on the similar list, ListChecker Pro 1.2 identified those same associations in the analysis of the dissimilar list.

The goal of ListChecker Pro 1.2 is to identify these lurking associations to help the researcher create the most "clean" lists possible for their experimental purposes. The experience of the author has been that creating lists of completely unrelated (no direct associations) cues and targets (and among cues/targets and other words on their lists) is entirely possible. Although the author has not attempted a list with no indirect associations, she has regularly created lists that controlled for indirect associations between words - for instance, allowing no more than two shared associates or mediators between any given cue-target pair-and for allowing a difference of no greater than .5 between lists (e.g., List 1 vs. List 2; Eakin \& Hertzog, 2006) or experimental conditions (e.g., control vs. interference; Eakin, 2005).

The purpose of the present article was to demonstrate that ListChecker Pro 1.2 can facilitate list construction in a variety of research areas. Although an example was used from the associative versus semantic priming literature, the intention is not to enter that theoretical discussion. Rather, the intention was to demonstrate that the bestintentioned list construction can sometimes result in less than optimal materials. The best scenario is to have knowledge as complete as possible about the characteristics of words and the relationships among them, whether direct or indirect. Indeed, the indirect associations may have a greater impact on experimental outcomes such as memory and metamemory than do direct associations (D. L. Nelson, personal communication, January 16, 2010). Indirect associations are not determined by small restricted preexperimental associative norming tasks; however, these associations have an impact on memory, metamemory, priming, visual word recognition, and other experimental outcomes. The USF word association norms provide information about both direct and indirect associations, and ListChecker Pro 1.2 is intended to be a helpful tool for checking created materials so that these characteristics are measured, controlled for, or manipulated.

\section{AUTHOR NOTE}

The author thanks Thomas Schreiber and Douglas L. Nelson for their contributions, conceptualizations, and edits of earlier forms of the manuscript. The author also thanks the reviewers and action editor for their thoughtful and careful comments and is grateful for the improvements to the manuscript due to their contributions. Correspondence should be directed to D. K. Eakin, Department of Psychology, Mississippi State University, P.O. Box 6161, Mississippi State, MS 39762 (e-mail: deakin@ psychology.msstate.edu)

\section{REFERENCES}

Collins, A. M., \& Loftus, E. F. (1975). A spreading-activation theory of semantic processing. Psychological Review, 82, 407-428. doi:10.1037/0033-295X.82.6.407

EAKIN, D. K. (2005). Illusions of knowing: Metamemory and memory under conditions of retroactive interference. Journal of Memory \& Language, 52, 526-534. doi:10.1016/j.jml.2005.01.009

EAKIN, D. K., \& Hertzog, C. (2006). Release from implicit interference in memory and metamemory: Older adults know that they can't let go. Journals of Gerontology, 61B, P340-P347.

Eakin, D. K., \& Hertzog, C. (2010a). Age invariance in feeling of knowing during implicit interference effects. Manuscript submitted for publication.

EAKIn, D. K., \& Hertzog, C. (2010b). Metamemory predictions at encoding are insensitive to implicit interference effects at retrieval. Manuscript submitted for publication.

FISCHLER, I. (1977). Semantic facilitation without association in a lexical decision task. Memory \& Cognition, 5, 335-339.

FlaVell, J. H. (1979). Metacognition and cognitive monitoring: A new area of cognitive-developmental inquiry. American Psychologist, 34, 906-911. doi:10.1037/0003-066X.34.10.906

Hertzog, C., Kidder, D. P., Powell-Moman, A., \& Dunlosky, J. (2002). Aging and monitoring associative learning: Is monitoring accuracy spared or impaired? Psychology \& Aging, 17, 209-225. doi:10.1037/0882-7974.17.2.209

Hutchinson, T. P. (2003). A notation for the structure of cognitive processes, with application to patterns of verb-processing impairment. Language \& Cognitive Processes, 18, 165-174. doi:10.1080/01690960143000506

LuCAS, M. (2000). Semantic priming without association: A metaanalytic review. Psychonomic Bulletin \& Review, 7, 618-630.

McClelland, J. L. (1987). The case for interactionism in language processing. In M. Coltheart (Ed.), Attention and performance XII: The psychology of reading (pp. 3-36). Hillsdale, NJ: Erlbaum.

McRae, K., \& Boisvert, S. (1998). Automatic semantic similarity 
priming. Journal of Experimental Psychology: Learning, Memory, \& Cognition, 24, 558-572. doi:10.1037/0278-7393.24.3.558

NeelY, J. H. (1991). Semantic priming effects in visual word recognition: A selective review of current findings and theories. In D. Besner \& G. W. Humphreys (Eds.), Basic processes in reading: Visual word recognition (pp. 264-336). Hillsdale, NJ: Erlbaum.

Nelson, D. L., Bennett, D. J., \& Leibert, T. W. (1997). One step is not enough: Making better use of association norms to predict cued recall. Memory \& Cognition, 25, 785-796.

Nelson, D. L., \& McEvoy, C. L. (1979). Encoding context and set size. Journal of Experimental Psychology: Human Learning \& Memory, 5, 292-314. doi:10.1037/0278-7393.5.3.292

Nelson, D. L., McEvoy, C. L., \& Dennis, S. (2000). What is free association and what does it measure? Memory \& Cognition, 28, 887899

Nelson, D. L., McEvoy, C. L., \& Pointer, L. (2003). Spreading activation or spooky action at a distance? Journal of Experimental Psychology: Learning, Memory, \& Cognition, 29, 42-52. doi:10.1037/0278 $-7393.29 .1 .42$

Nelson, D. L., McEvoy, C. L., \& Schreiber, T. A. (1990). Encoding context and retrieval conditions as determinants of the effects of natural category size. Journal of Experimental Psychology: Learning, Memory, \& Cognition, 16, 31-41. doi:10.1037/0278-7393.16.1.31

Nelson, D. L., McEvoy, C. L., \& Schreiber, T. A. (1998). The University of South Florida word association, rhyme, and word fragment norms. Available at http://web.usf.edu/FreeAssociation.

Nelson, D. L., McEvoy, C. L., \& Schreiber, T. A. (2004). The University of South Florida free association, rhyme, and word fragment norms. Behavior Research Methods, Instruments, \& Computers, 36, 402-407.

Nelson, D. L., McKinney, V. M., Gee, N. R., \& Janczura, G. A. (1998). Interpreting the influence of implicitly activated memories on recall and recognition. Psychological Review, 105, 299-324.

Nelson, D. L., Schreiber, T. A., \& McEvoy, C. L. (1992). Processing implicit and explicit representations. Psychological Review, 9, 322348. doi:10.1037/0033-295X.99.2.322

Nelson, D. L., Schreiber, T. A., \& Xu, J. (1999). Cue set size effects: Sampling activated associates or cross-target interference? Memory \& Cognition, 27, 465-477.
Nelson, D. L., \& Zhang, N. (2000). The ties that bind what is known to the recall of what is new. Psychonomic Bulletin \& Review, 7, 604617.

Pécher, D., Zeelenberg, R., \& Raaijmakers, J. G. W. (1998). Does PIZZA prime COIN? Perceptual priming in lexical decision and pronunciation. Journal of Memory \& Language, 38, 401-418. doi:10.1006/ jmla.1997.2557

Plaut, D. C., \& Boоth, J. R. (2000). Individual and developmental differences in semantic priming: Empirical and computational support for a single-mechanism account of lexical processing. Psychological Review, 107, 786-823. doi:10.1037/0033-295X.107.4.786

SCHREIBER, T. A. (1998). Effects of target set size on feelings of knowing and cued recall: Implications for the cue effectiveness and partialretrieval hypotheses. Memory \& Cognition, 26, 553-571.

Schreiber, T. A., \& Nelson, D. L. (1998). The relation between feelings of knowing and the number of neighboring concepts linked to the test cue. Memory \& Cognition, 26, 869-883.

Steyvers, M., \& Tenenbaum, J. B. (2005). The large-scale structure of semantic networks: Statistical analyses and a model of semantic growth. Cognitive Science, 29, 41-78. doi:10.1207/s15516709 $\operatorname{cog} 2901 \_3$

WILLIAMS, J. N. (1996). Is automatic priming semantic? European Journal of Cognitive Psychology, 8, 113-161.

Yates, M., Locker, L., JR., \& Simpson, G. B. (2003). Semantic and phonological influences on the processing of words and pseudohomophones. Memory \& Cognition, 31, 856-866.

\section{NOTES}

1. The University of South Florida word association, rhyme, and word fragment norms (Nelson, McEvoy, \& Schreiber, 1998, 2004) were developed to measure and catalogue both individual and associative characteristics of words. A complete description and the database are available elsewhere and are not reproduced in the present article.

2. For more details on calculating mediator and shared associate strength, see Nelson et al. (2004).

(Manuscript received February 15, 2010; revision accepted for publication June 13, 2010.) 